\title{
Forced convection in a variable section axisymmetric channel with different porous layers and heat generation
}

\author{
E. Pilevne \& A. Misirlioglu \\ Faculty of Aeronautics and Astronautics, Istanbul Technical University, \\ Maslak, 34469, Istanbul, Turkey
}

\begin{abstract}
In the present study, the forced convection in an axisymmetric channel has been investigated numerically. The channel, which contains different porous layers, has variable cross sectional areas along the axis. The governing equations for non-Darcy porous media are solved for the uniform inlet velocity profile and uniform inlet temperature. The walls are kept at a constant temperature. The porous medium at the middle of the channel has internal heat generation. This kind of solution domain is aimed to model the phenomena in the porous burners. The finite element method is employed to solve the governing equations. First, the code is compared for the fully developed flow in the parallel channel. For this purpose the maximum velocities at the channel axis and the Nusselt numbers at the wall are compared with the literature. Having obtained the validated results for the code, it is applied to the problem described above. The results will be presented in terms of velocity and temperature profiles.
\end{abstract}

Keywords: porous layers, forced convection, FEM.

\section{Introduction}

Applications of porous media in engineering have constantly been growing in last two decades. In particular, the heat transfer characteristics of the porous medium is of high importance due to the applications in, for example, heat exchangers, transport of heated or cooled fluids, micro-electronic cooling, chemical processing equipment, and porous burners, etc. Apart from the conventional burners, porous media burners provide many advantages in especially industrial applications. Within this technology it is able to build more 
basic combustion chamber configurations and the size of the porous burner can be diminished by approximately $10 \%$ compared to burners with conventional shapes. In addition, low emission rates and constitution of stable combustion have lately been making this technology more desirable. The porous burners consist of three porous layers with different Péclet numbers. The main purpose of using different layers is to control the heat transfer. The aim of the first porous layer is having a uniform velocity profile before the ignition phase. The ignition process is carried out by the second layer. Within the third layer flame propagation is established. Research and development on combustion in porous media started to grow in last two decades. Trimis [1] showed that porous media can be very useful within many applications in energy and heat-engineering. Bassam and Abu-Hijleh [2] studied heat transfer from a 2D backward facing step with different porous segments and also they analyzed the effect of these layers on local and overall Nusselt numbers. Furthermore, Nield et al. [3] examined the interaction of two porous layers with the same porosity and permeability but different thermal conductivity effects in forced convection. Nemoda et al. [4] simulated a porous burner and surface burner numerically with different heat conductivity and power of burners. Another studies are the effects of material and radiative properties on flame stabilization in a porous burners [5, 6]. Mishra et al. analyzed the heat transfer of a radiant porous burner [7]. The main purpose of this paper is to investigate the effect of Peclet number and permeability on the heat transfer in forced convection. The geometry is designed as having variable cross sections and axisymmetric. The wall is kept at constant temperatures. The numerical model is obtained using Navier-Stokes with modified Darcy's law. The Galerkin Finite Elements method is applied to solve the governing equations. A source term is added into the energy equation in order to simulate the heat generation in the porous layer.

\section{Formulation}

The solution domain and the boundary conditions are shown in Figure 1, where the uniform flow enters into the axisymmetric duct with variable section radius, and with different properties. The inlet and expansion regions are considered as filled with clear fluid. Then the flow passes thru porous layers of 3 different permeabilities. The middle porous section has heat generation inside. The governing equations written in terms of the volume averaged intrinsic velocities, for uniform property distribution in each section, are [8]:

Continuity

Momentum

$$
\nabla \cdot \mathbf{u}=0
$$

$$
\frac{D \mathbf{u}}{D t}=-\nabla p+\frac{1}{\mathrm{Re}} \nabla^{2} \mathbf{u}-\frac{\varepsilon}{\mathrm{ReDa}_{\mathrm{i}}} \mathbf{u}
$$

Energy

$$
\frac{D \theta}{D t}=\frac{1}{\mathrm{Pe}_{\mathrm{i}}}\left[\nabla^{2} \theta+\lambda\right]
$$




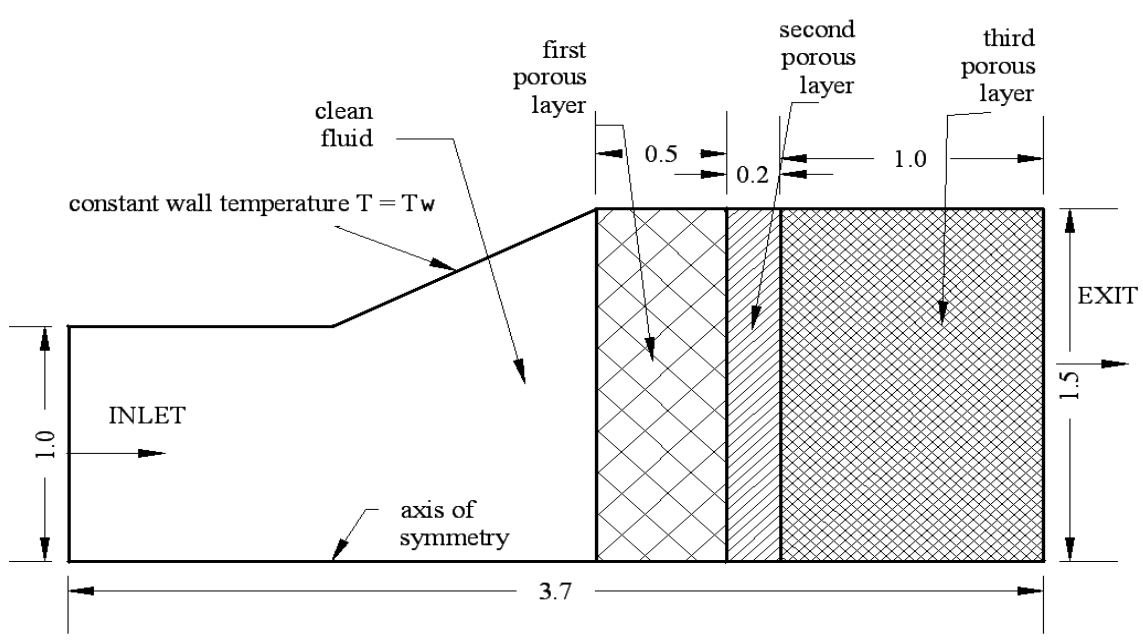

Figure 1: Solution domain and boundary conditions.

The derivative operators in these equations are evaluated in polar coordinates. The following parameters are used to obtain the above dimensionless governing equations:

$$
\begin{array}{ll}
x=\bar{x} / R \quad y=\bar{y} / R & t=\left(U_{\infty} / R\right) \bar{t} \quad \mathbf{u}=\overline{\mathbf{u}} / U_{\infty} \\
\theta=\left(T-T_{W}\right) / \Delta T & \Delta T=q_{m}^{\prime \prime \prime} R^{2} / k_{m}
\end{array}
$$

where $T_{W}$ is wall temperature, $R$ is the channel radius at inlet, $U_{\infty}$ is the inlet velocity. In these equations, $\mathrm{Da}_{\mathrm{i}}$ and $\mathrm{Pe}_{\mathrm{i}}$ are the Darcy and Peclet numbers of the clear fluid and porous insert regions defined as

$$
\mathrm{Da}_{\mathrm{i}}=\frac{\mathrm{K}_{\mathrm{i}}}{R^{2}} \quad \mathrm{Pe}_{\mathrm{i}}=\operatorname{RePr}_{\mathrm{i}} \quad \mathrm{i}=1 \text {, number of regions }
$$

where $K_{i}$ 's are the permeabilities of each porous insert, Re is the Reynolds number based on the uniform inlet velocity and the inlet radius, $\mathrm{Pr}_{i}$ 's are the Prandtl numbers for each region. $\lambda$ takes the value of 1 if there is heat source in the region, otherwise zero.

The flow in the channel is driven by the uniform inlet velocity profile. At the exit of the channel the flow is assumed to be parallel, and at the duct wall no-slip condition is applied. The temperatures are assumed to be equal at the inlet and the duct wall. Symmetry and exit conditions are imposed on the proper boundaries.

\section{Numerical treatment}

Galerkin Finite Element method is employed to solve the governing equations (1)-(3) subjected to the given boundary conditions. The Fractional Step method is used for the time integration and for the calculation of pressure, based on the 
technique suggested by Kovacs and Kawahara [11]. The solution domain of 4 different types of region is evaluated as a single domain. Property changes for the regions are included at the element level and the interface nodes are interpolated as other properties by the finite element method itself. By doing this, there is no need to impose any compatibility boundary condition on the interfaces of neighbouring regions. The temperature distribution is observed at consecutive time steps to check if the solution has reached to the steady state or not.

\section{Results}

There are four different types of region and each of them has been defined in calculations with different Péclet and Darcy numbers. The Reynolds number is taken as 100. The Péclet number is held in the first porous layer 50. For the following layers it is taken 65 and 70 . There are 4000 points in the solution domain. The grid size varies 0.02 near the walls and expands to 0.1 in the midsection of the channel. Time step is chosen 0.001 to satisfy a stable solution.

The code used in the present study is validated with the results of Vafai and Kim [10] for fully developed flow in parallel channel in terms of velocity profile and with Kaviany [8] for the Nusselt numbers. As seen in Figure 2, the results are in perfect agreement with the literature.
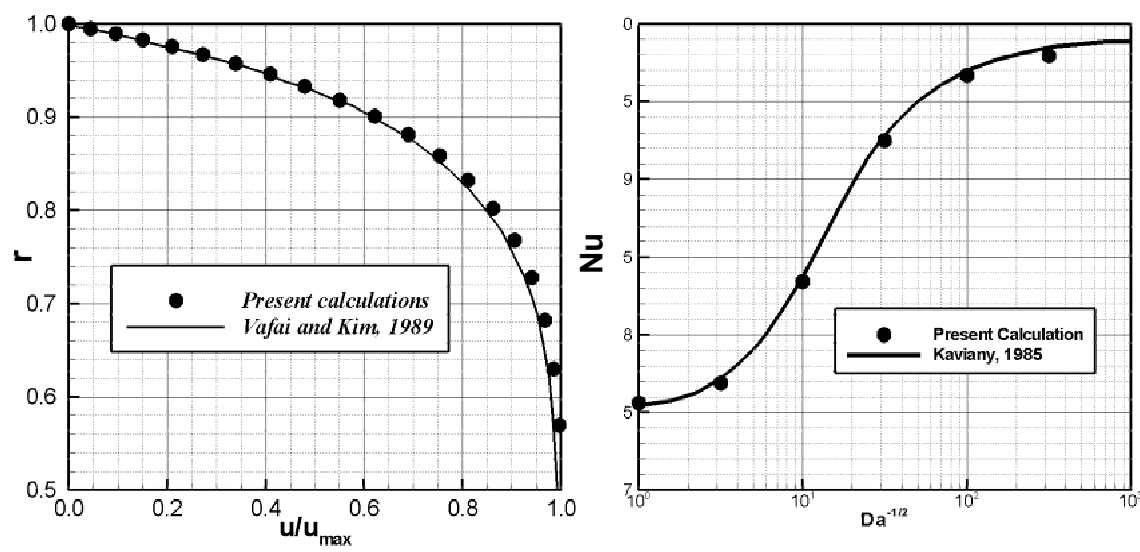

Figure 2: Comparison of the velocity profile (left) for $\mathrm{Da}=10^{-2}$ and Nusselt numbers (right) obtained for a parallel channel.

Obtaining validated results the code is employed to the problem specified before for 3 different cases.

- Case 1: the duct is filled with clear fluid, no porous region exists

- Case 2: the duct has clear fluid region at the entrance and expansion regions, and the rest of the duct is filled with porous media with the same permeability for Darcy number of $10^{-4}$. 
- Case 3: the porous media in Case 3 has been divided into 3 regions with different permeabilities corresponding to Darcy numbers which are $10^{-3}, 10^{-4}, 10^{-5}$ in order.

The results for these cases are presented in exit velocity and temperature profiles (Figure 3), and temperature at the axis (Figure 4), streamlines (Figure 5), and temperature contours (Figure 6).
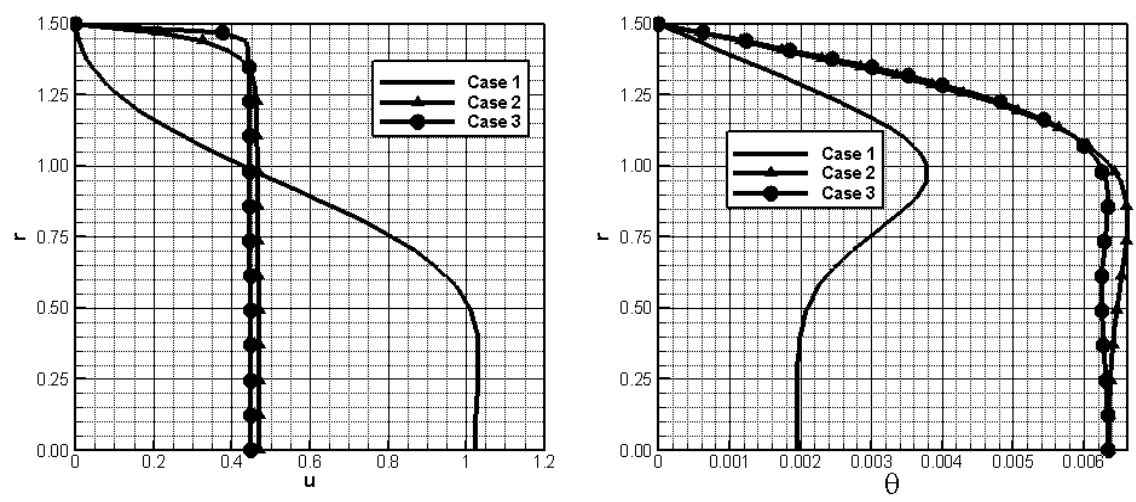

Figure 3: Velocity (left) and temperature (right) profiles at the exit for all cases.

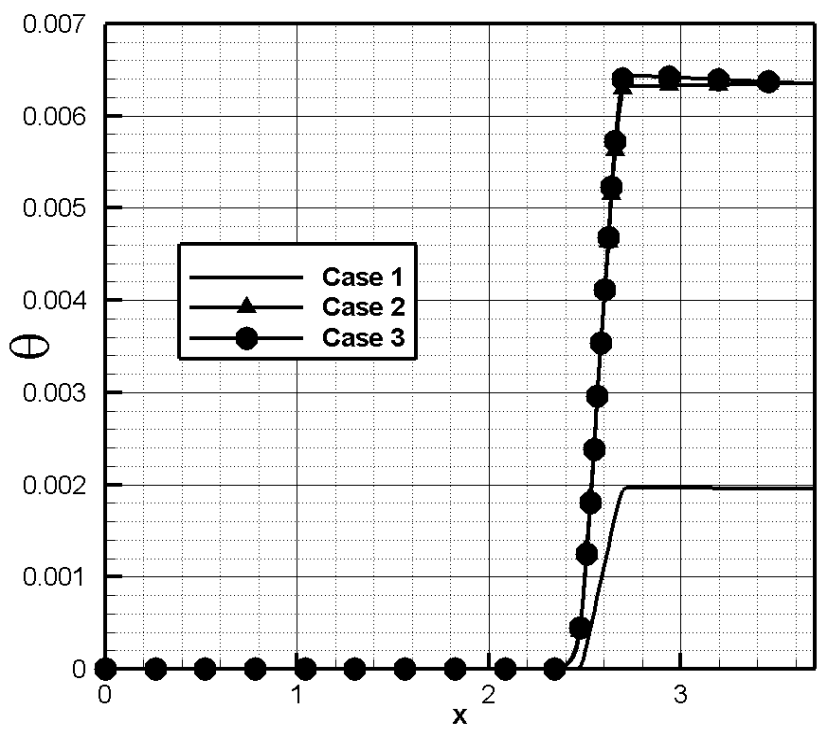

Figure 4: Temperature along the axis of the duct. 


\section{Case 1}

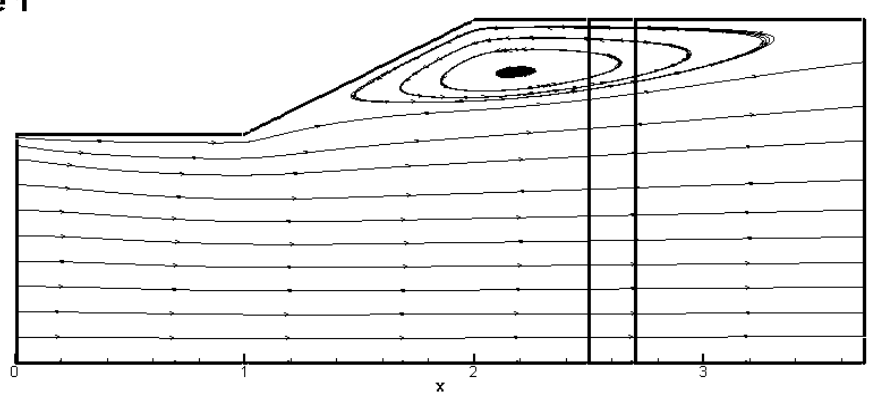

Case 2

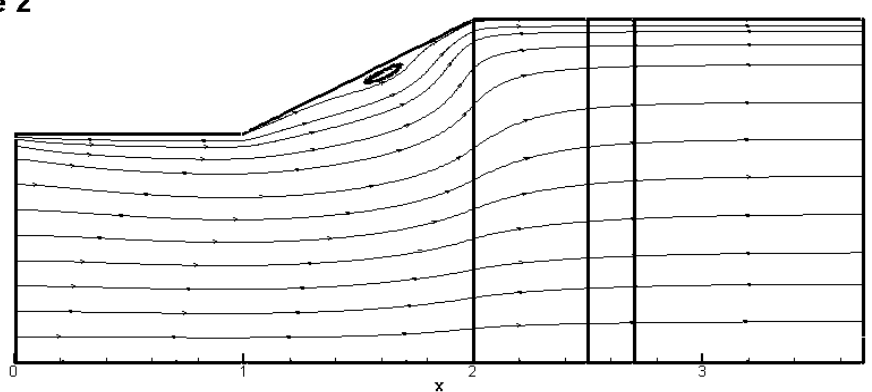

\section{Case 3}

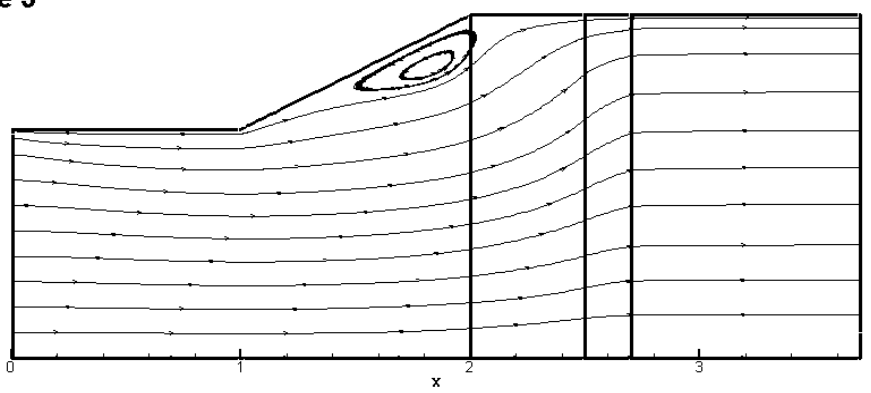

Figure 5: $\quad$ Streamlines for all cases.

In Figure 3 the effect of the porous media is clearly seen by the uniformity of the velocity distribution at the exit. This also affects the temperature distribution at the exit, and makes it more uniform. For Cases 2 and 3 although there is a negligible difference in the velocity profiles, the difference in temperature profiles is much clearer. Using different permeabilities provide more heat to discharge from the domain. Another aspect is the increment of the temperature along the symmetry axis, as seen in Figure 4 . The porous media in Cases 2 and 3 provides higher temperature increments. 
The main reason of differences between Case 1 and Cases 2 and 3 can be clearly seen in the flow and thermal fields in Figures 5 and 6. As seen in Figure 5 the flow in Case 1 separates after the expansion region, and makes a big bubble. This bubble prevents the heat generated in the duct to convect downstream. Thus a hot spot is formed in the duct as seen in Figure 6. But this kind of structure is not seen in the Cases 2 and 3. Prevention of flow separation provides better thermal distribution in the channel. The separation bubbles in case 2 and 3 have been formed in the expansion region, where no heat generation exists, and no separation region reaches to the porous layers.

\section{Case 1}

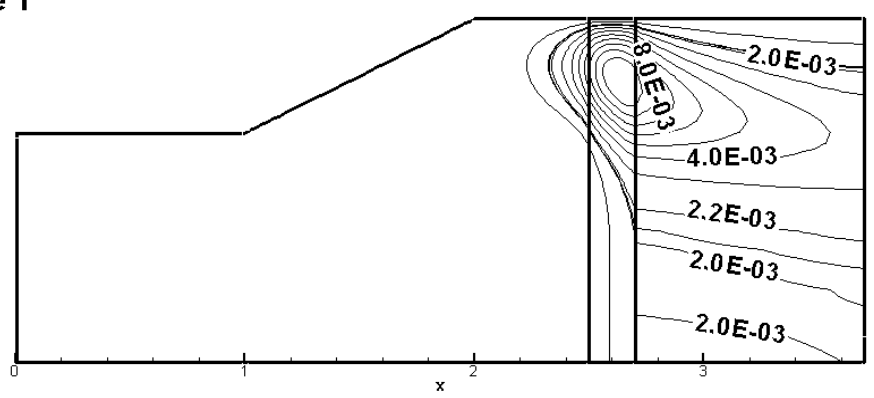

Case 2

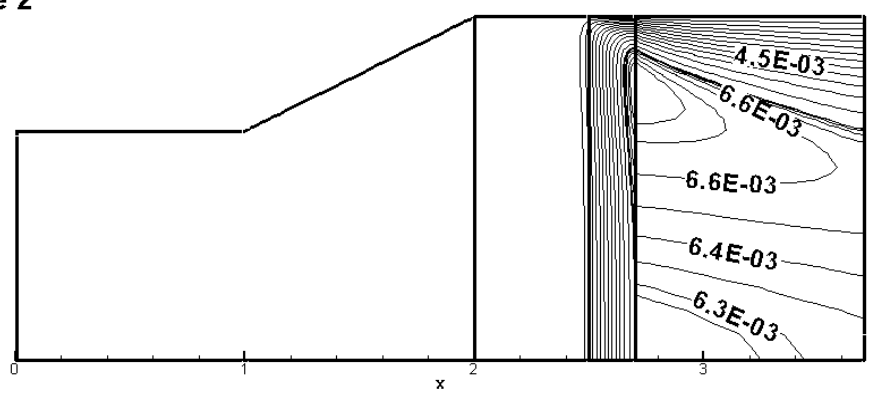

Case 3

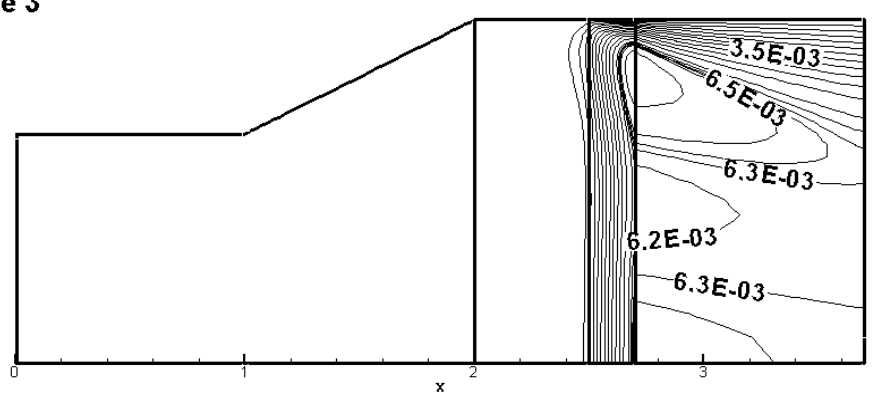

Figure 6: Temperature contours for all cases. 


\section{Conclusion}

The flow and heat character of an axisymmetric channel with variable cross sections has been investigated numerically. The effect of the porous media has been shown in terms of temperature and flow field variables. The porous medium has a stabilizing effect on the thermal structure, which results in more heat being discharged from the duct. When there is no porous medium in the channel the separation prevents the heat from to convecting in the channel. This effect can easily be removed by aerodynamic improvement of the channel inlet and expansion regions. For uniform permeability distribution (Case 2) in the channel and variable permeability distribution there is little change in the channel in terms of flow characteristics. However the thermal character changes and Case 3 provides better temperature distribution.

\section{References}

[1] Trimis D., Stabilized Combustion in Porus-Media-Applications of the Porous Burner Technology in Energy- and Heat-Engineering, Fluids 2000 Conference and Exhibit, Denver

[2] Bassam A., K. Abu-Hijleh, Heat transfer from a 2D backward facing step with isotropic porous floor segments, Int. J. Heat and Mass Transfer, 43, pp. 2727-2737, 2000.

[3] Nield, D.A., Kuznetsov, A.V., Xiong, M., Effect of heterogeneity in forced convection in a porous medium: parallel plate channel or circular duct, Int. J. Heat and Mass Transfer, 43, pp. 4119-4134, 2000.

[4] Stevan Nemoda, Dimosthenis Trimis, Goran Zivkovic, Numerical Simulation Of Porous Burners And Hole Plate Surface Burners, Thermal Science: 8(1), pp. 3-17, 2004

[5] Amanda J. Barraa, Guillaume Diepvensa, Janet L. Ellzeya, Michael R. Henneke, Numerical study of the effects of material properties on flame stabilization in a porous burner, Combustion and Flame 134, pp. 369-379, 2003.

[6] Isabel Malico, Jose Carlos F. Pereira, Numerical Study on the Influence of Radiative Properties in Porous Media Combustion, Journal of Heat Transfer, Vol. 123, pp. 951-957

[7] S.C. Mishra, M. Steven, S. Nemoda, P. Talukdar, D. Trimis, F. Durst, Heat transfer analysis of a two-dimensional rectangular porous radiant burner, International Communications in Heat and Mass Transfer 33, pp. 467-474, 2006

[8] Kaviany, M., Laminar flow through a porous channel bounded by isothermal parallel plates, Int. J. Heat and Mass Transfer, 28, pp. 851-858, 1985.

[9] Saeid H. N., Analysis of mixed convection in a vertical porous layer using non-equilibrium model, Int. J. Heat and Mass Transfer, 47, pp. 56195627, 2004. 
[10] Vafai K., Kim S.J., Forced Convection in a channel filled with a porous medium: An exact solution, Journal of Heat Transfer, 111, pp. 11031106,1989

[11] A. Kovacs, M. Kawahara, A Finite element scheme based on the velocity correction method for the solution of the time-dependent incompressible Navier-Stokes equations, Int. J. Numer. Methods Fluids, 13, pp. 403-423, 1991. 